\title{
Could combinations of new and old drugs enhance tumor cell death?
}

\author{
João MA Delou ${ }^{1}$, Karina L Hajdu ${ }^{1}$ \& Helena L Borges*,1 \\ ${ }^{1}$ Biomedical Sciences Institute, Federal University of Rio de Janeiro, Rio de Janeiro, 21949-590, Brazil \\ *Author for correspondence: Tel.: +55 21 39386467; hborges@icb.ufrj.br
}

\section{"Regardless of the mechanism for enhancing cancer cell death, high-throughput screening greatly facilitates the discovery of new uses for FDA approved 'old drugs' and selection of the next generation of anticancer chemotherapy."”}

First draft submitted: 30 April 2018; Accepted for publication: 3 August 2018; Published online: 24 August 2018 Keywords: anticancer chemotherapy $\bullet$ antineoplastic agents $\bullet$ cell cycle $\bullet$ cell death $\bullet$ chemotherapy $\bullet$ clinical trials
$\bullet$ cyclin-dependent kinases $\bullet$ drug combinations $\bullet$ drug development $\bullet$ polypharmacology

The concept of anticancer combination therapy began when E Frei, JF Holland and EJ Freireich published the first comparative study of antileukemic drug combination in children with acute lymphocytic leukemia [1]. Since then, numerous studies and clinical trials made combination therapy the gold standard in today's cancer chemotherapy. Unfortunately, the initial hope that multiple combinations would eradicate all cancer cell population in all patient, has not yet become tangible. The National Cancer Institute lists over 250 anticancer drug information summaries including common combinations for each cancer type. However, beside this apparently long list of treatment choices, many of the listed drugs are aimed to alleviate the symptoms or severe side effects induced by the anticancer treatment, such as cardiac-, liver-, renal- and neuro-toxicity, as well as pain, diarrhea, fatigue, neutropenia and many others. Therefore, the design of new and more effective treatments, through the search for new selective drugs, multitarget or repurposed medications has never been as actively pursued as today.

Repurposing studies have been in the spotlight of pharmaceutical R\&D searching for new uses of medications that are already approved by the US FDA for some years now [2]. Even if the FDA has not approved a drug for one use yet, an off-label drug may become accepted by the medical community and widely used as treatment often faster than its regulation. Many studies have shown that old drugs can be applied successfully to anticancer therapy, either as a single agent, or in combination with classical chemotherapy or even together with new drugs. Several of these combinations of old drugs with new ones are being tested in clinical trials, and some will be highlighted here.

Although some widespread combinations are FDA approved, such as folinic acid, fluorouracil and oxaliplatin (FOLFOX) for colorectal cancer and procarbazine hydrochloride, lomustine, also known as CCNU, and vincristine sulfate (PCV) for gliomas, many used combinations might not be approved since it might not be factual for the FDA to approve each one of them. Nevertheless, it is much cheaper to propose new combinations to treat cancer when detailed information is available on their pharmacology, formulation and potential toxicity.

One example of an old drug under repurposing studies is olanzapine. It is a multitarget second-generation antipsychotic medication used to treat bipolar disorder and schizophrenia that seems to act both as dopamine D2 and serotonin $5-\mathrm{HT}_{2 \mathrm{~A}}$ receptors antagonist. Sanomachi et al. have recently presented its proapoptotic antisurvivin effect on lung and pancreatic cancer stem cell lines and its sensitizing effect to old chemotherapeutic agents such as cisplatin, 5-fluorouracil (5-FU) and gemcitabine in a survivin expression-dependent manner [3].

Another old drug with anticancer potential recently proposed to be repositioned to anticancer chemotherapy is the antifungal tioconazole. Liu et al. have shown that tioconazole inhibited ATG4 and autophagy in glioblastoma, colorectal and breast carcinoma in spheroid cell culture and in colorectal HCT116 xenografted mice leading to enhanced doxorubicin drug-induced cytotoxicity [4]. In fact, several other chemotherapeutic agents are known to modulate autophagy and consequently blocking autophagic flux to increase cancer cell death [5-7]. 
Two of the first autophagy modulators described, and which have been already proposed to be repositioned for cancer treatment, are the antimalarials chloroquine and its related drug hydroxychloroquine. They are both autophagy inhibitors that have been extensively studied in vitro and in vivo as possible agents against several solid cancers (reviewed in [7]).

There has been only one Phase III cohort study with chloroquine for cancer treatment. It was an international cooperative Phase III trial of randomized, placebo-controlled, double-blind study of the effects of chloroquine in adjuvancy to the conventional therapy of glioblastoma (NCT00224978). Since the publication of its promising results in 18 patients with glioblastoma in 2003, several authors point to the need for more and larger trials. These initial results were complemented by another publication in 2006, where 30 patients with surgically confirmed glioblastoma received oral chloroquine for 12 months. Although the small sample hampered an independent analysis of confounding factors, the results were favorable to the chloroquine treated group, which had an improvement in mid-term survival when given in combination to conventional chemotherapy. Therefore, a positive conclusion in favor of more and larger Phase III trials was given [8].

Several Phase I and II clinical trials of chloroquine and hydroxychloroquine have been conducted in several solid tumors, such as lung, breast, melanoma, colorectal, pancreatic and glioma/glioblastoma (reviewed in [7]), but there is no registry of another Phase III cohort yet.

Regardless of the mechanism for enhancing cancer cell death, either by blocking of the autophagic flux or other therapeutic pathways, high-throughput screening greatly facilitates the discovery of new uses for FDA approved 'old drugs' and selection of the next generation of anticancer chemotherapy.

Besides repurposing studies, several new pharmacological classes aimed to interfere with obvious targets in the control of cell cycle, DNA repair and survival pathways are under development. Some of these drugs have already been approved for cancer treatment, such as PI3K, PARP and CDKs inhibitors. Cell cycle modulation can increase the anticancer cytotoxicity of several old chemotherapeutic agents such as platins, taxanes, anthracyclines or nucleoside analogs. Cisplatin and doxorubicin cytotoxicity, for example, is enhanced by depletion of cell cycle checkpoint control proteins, such as ATM and CHK2, concomitant to deficiency of the tumor suppressor TP53 on breast cancer cells [9]. Another example of cell cycle modulation is the inhibition of WEE1 by AZD1775, that synergizes with genotoxic agents (gemcitabine, carboplatin or cisplatin) in several TP53-deficient cells, such as colorectal, non-small cell lung, ovarian and pancreatic human carcinomas [10]. Recently, Soletti et al. (2017) showed that CDK inhibition also enhances cisplatin cytotoxicity in esophageal cancer cell lines [11].

CDKs, especially CDK4 and 6, are crucial regulators of the cell cycle, which are physiologically inhibited by p16/INK4 during interphase. Loss of p16/INK4 and/or gene amplification of CDK4/ 6 and their corresponding cyclins is a common feature of several cancer types. The inhibition of these CDKs is thought to be a preferable target in anticancer therapy because they act downstream of many other mitogenic signals, therefore mitigating the risk of development of compensatory mechanisms [12].

The first studies targeting CDK inhibition evaluated pan-CDK inhibitors such as flavopiridol (Sanofi Aventis, Îlede-France, France), which inhibits CDKs -1, -2, -4, -6, -7 and -9. Clinical trials with these pan-CDK inhibitors failed to show them as beneficial as expected. Their little therapeutic effects and high dose-limiting toxicity rates [13], mainly attributed to off-target interactions, lead to the discontinuity of their Phase II studies. In more recent years, three CDK4/6 specific inhibitors were successfully developed and FDA-approved, namely palbociclib (PD0332991, Pfizer, NY, USA), ribociblib (LEE011, Novartis, Basel, Switzerland) and abemaciclib (LY2835219, Eli Lilly, Indianapolis, USA).

Palbociclib was approved as first-line treatment for hormone receptor- (HR)-positive and HER2-negative advanced breast cancer patients in combination with the aromatase inhibitor letrozole. The PALOMA-1/TRIO-18 Phase II study showed a progression-free survival of 20.2 months in the palbociclib group (84 patients) against 10.2 months for the letrozole group ( 81 patients, HR: 0.488, 95\% CI: $0.319-0.748$; one-sided p $=4 \times 10^{-4}$ ) [14].

Ribociclib was also approved as first-line treatment for the same breast cancer group in 2016, after MONALEESA2 trial showed significant improvements in progression-free survival and higher rates of clinical benefits for patients who received ribociclib in combination with letrozole (334 patients) when compared to the letrozole plus placebo group (334 patients; HR: 0.556, 95\% CI: 0.429-0.720; $\mathrm{p}=3.29 \times 10^{-6}$ ) [15].

Abemaciclib received its approval in September 2017, either as monotherapy or in combination with the selective estrogen receptor degrader fulvestrant, for HR-positive/HER2-negative advanced or metastatic breast cancer, for patients presenting disease progression after endocrine therapy. MONARCH-3 trial showed increased progression- 
free survival between 328 patients who received abemaciclib (28.2 months) plus chemotherapy against 165 patients in the placebo plus chemotherapy group (14.8 months; HR: 0.540, 95\% CI: $\left.0.418-0.698 ; \mathrm{p}=2.1 \times 10^{-5}\right)[16]$.

For now, CDK4/6 inhibitors have shown successful results for breast cancer with efforts being made to develop treatment strategies against its other molecular subtypes. One of the main issues for chemotherapy is acquired resistance. In a study by McClendon et al., palbociclib hampered doxorubicin cytotoxicity in breast cancer cells, in a pathway dependent on diminished cleaved PARP and E2F1 levels, antagonize drugs that are dependent on cell cycle progression, such as anthracyclines avoiding progression of the cell cycle. Therefore, it emphasizes the growing notion that CDK4/6 inhibitors might antagonize drugs that are dependent on cell cycle progression, and the order in which the drugs are given might affect treatment efficiency and resistance $[17,18]$.

There is a growing number of preclinical studies looking at the use of combination therapy with CDK4/6 inhibitors in other cancer types. For example, in pancreatic cancer cells, Franco et al. showed that the combination of palbociclib with drugs that act on proliferation and cell division might show either sinergic or antagonic effects, depending on the stage of the cell cycle affected. For instance, the combination with taxanes and PLK1 inhibitors, which both act on cell division, showed antagonic effects. Meanwhile, the combination with 5-FU or MEK inhibitors, which both act on G1/S transition, produces a slightly cooperative interaction, increasing cancer cell death [18].

The G1/S transition seems to be a particularly important target of treatment with CDK inhibitors in several solid cancers. There is a lack of research on combination chemotherapy on solid cancers other than breast, where CDK inhibitors are already more advanced. This was recently reviewed elsewhere [19].

One example of these few studies showed that palbociclib and irinotecan combination in colorectal cancer cell lines produced increased cytotoxicity due to higher caspase-3 activation [20].

In summary, regardless of the mechanism for enhancing cancer cell death, either for blocking autophagy or death pathways, the discovery of new uses for FDA approved 'old drugs' for increasing the compendium of anticancer treatments is urgent. Although CDK4/6 inhibitors are very promising drugs for cancer therapy, further investigation is necessary to determine for which types of cancers they are effective, besides the initial FDA approved use and also in which combinations they are most beneficial. Aiming for different molecular pathways that are altered in specific types of cancers might be the answer to increase efficacy with less side effects. In this scenario, high throughput screening for selecting synergistic combinations of new drugs, including 'new old drugs', might be the quickest possible transition from bench to bedside. Hopefully, in the near future, it might be possible to design the individual best combination approach to improve cancer treatment and minimize patient suffering.

Acknowledgements

Assistance by D Biasoli in reviewing the manuscript was greatly appreciated.

Financial \& competing interests disclosure

The authors thank the National Council for Scientific and Technological Development (CNPq), and Rio de Janeiro Research Foundation (FAPERJ) for financial support. The authors have no other relevant affiliations or financial involvement with any organization or entity with a financial interest in or financial conflict with the subject matter or materials discussed in the manuscript apart from those disclosed.

No writing assistance was utilized in the production of this manuscript.

\section{References}

Papers of special note have been highlighted as: $\bullet$ of interest; $\bullet \bullet$ of considerable interest

1. Frei E 3rd, Karon M, Levin RH et al. The effectiveness of combinations of antileukemic agents in inducing and maintaining remission in children with acute leukemia. Blood 26(5), 642-656 (1965).

-. First ever comparative study of anticancer drug combinations in humans.

2. Nosengo N. Can you teach old drugs new tricks? Nature 534(7607), 314-316 (2016).

3. Sanomachi T, Suzuki S, Kuramoto K et al. Olanzapine, an atypical antipsychotic, inhibits survivin expression and sensitizes cancer cells to chemotherapeutic agents. Anticancer Res. 37(11), 6177-6188 (2017).

- A well-written commentary on current drug repurposing studies.

4. Liu PF, Tsai KL, Hsu CJ et al. Drug repurposing screening identifies tioconazole as an ATG4 inhibitor that suppresses autophagy and sensitizes cancer cells to chemotherapy. Theranostics 8(3), 830-845 (2018).

- Recent identification of a well-known and safe antifungal drug as adjuvant in anticancer chemotherapy. 
5. Biasoli D, Kahn SA, Cornelio TA et al. Retinoblastoma protein regulates the crosstalk between autophagy and apoptosis, and favors glioblastoma resistance to etoposide. Cell Death Dis. 4, e767 (2013).

6. Delou JM, Biasoli D, Borges HL. The complex link between apoptosis and autophagy: a promising new role for RB. An. Acad. Bras. Cienc. 88(4), 2257-2275 (2016).

7. Marinkovic M, Sprung M, Buljubasic M, Novak I. Autophagy modulation in cancer: current knowledge on action and therapy. Oxid. Med. Cell Longev. 2018, 8023821 (2018).

- Well-written review on the clinical benefits of autophagy modulation in cancer.

8. Sotelo J, Briceno E, Lopez-Gonzalez MA. Adding chloroquine to conventional treatment for glioblastoma multiforme: a randomized, double-blind, placebo-controlled trial. Ann. Intern. Med. 144(5), 337-343 (2006).

9. Jiang H, Reinhardt HC, Bartkova J et al. The combined status of ATM and p53 link tumor development with therapeutic response. Genes Dev. 23(16), 1895-1909 (2009).

10. Hirai H, Iwasawa Y, Okada M et al. Small-molecule inhibition of Wee1 kinase by MK-1775 selectively sensitizes p53-deficient tumor cells to DNA-damaging agents. Mol. Cancer Ther. 8(11), 2992-3000 (2009).

11. Soletti RC, Biasoli D, Rodrigues $\mathrm{N}$ et al. Inhibition of $\mathrm{pRB}$ pathway differentially modulates apoptosis in esophageal cancer cells. Transl. Oncol. 10(5), 726-733 (2017).

12. Knudsen ES, Witkiewicz AK. The strange case of CDK4/6 inhibitors: mechanisms, resistance, and combination strategies. Trends Cancer 3(1), 39-55 (2017).

- Great review of CDK4/6 inhibitors development.

13. Xu H, Yu S, Liu Q et al. Recent advances of highly selective CDK4/6 inhibitors in breast cancer. J. Hematol. Oncol. 10(1), 97 (2017).

14. Finn RS, Crown JP, Lang I et al. The cyclin-dependent kinase 4/6 inhibitor palbociclib in combination with letrozole versus letrozole alone as first-line treatment of oestrogen receptor-positive, HER2-negative, advanced breast cancer (PALOMA-1/TRIO-18): a randomised Phase 2 study. Lancet Oncol. 16(1), 25-35 (2015).

-• First clinical trial to take a CDK inhibitor to US FDA approval as first-line chemotherapy.

15. Hortobagyi GN, Stemmer SM, Burris HA et al. Ribociclib as first-line therapy for HR-positive, advanced breast cancer. N. Engl. J. Med. 375(18), 1738-1748 (2016).

16. Goetz MP, Toi M, Campone M et al. MONARCH 3: abemaciclib as initial therapy for advanced breast cancer. J. Clin. Oncol. 35(32), 3638-3646 (2017).

17. McClendon AK, Dean JL, Rivadeneira DB et al. CDK4/6 inhibition antagonizes the cytotoxic response to anthracycline therapy. Cell Cycle 11(14), 2747-2755 (2012).

18. Franco J, Witkiewicz AK, Knudsen ES. CDK4/6 inhibitors have potent activity in combination with pathway selective therapeutic agents in models of pancreatic cancer. Oncotarget 5(15), 6512-6525 (2014).

19. Vijayaraghavan S, Moulder S, Keyomarsi K, Layman RM. Inhibiting CDK in cancer therapy: current evidence and future directions. Target Oncol. 13(1), 21-38 (2018).

20. Zhang J, Zhou L, Zhao S, Dicker DT, El-Deiry WS. The CDK4/6 inhibitor palbociclib synergizes with irinotecan to promote colorectal cancer cell death under hypoxia. Cell Cycle 16(12), 1193-1200 (2017). 